\title{
Correlation between Mental Health and Traditional Chinese Medicine Constitutions: An Analysis Based on a Sample from a Tertiary Hospital Female Nurses of China
}

\author{
Jihui Yue, Xiaojuan Fang, Minfeng Cheng* \\ Department of Psychology, Fifth Affiliated Hospital, Sun Yat-sen University, Zhuhai, China \\ Email: *emin7641@163.com
}

How to cite this paper: Yue, J.H., Fang, X.J. and Cheng, M.F. (2019) Correlation between Mental Health and Traditional Chinese Medicine Constitutions: An Analysis Based on a Sample from a Tertiary Hospital Female Nurses of China. Open Journal of Social Sciences, 7, 220-229. https://doi.org/10.4236/jss.2019.79016

Received: August 19, 2019

Accepted: September 21, 2019

Published: September 24, 2019

Copyright $\odot 2019$ by author(s) and Scientific Research Publishing Inc. This work is licensed under the Creative Commons Attribution International License (CC BY 4.0).

http://creativecommons.org/licenses/by/4.0/

\section{(c) (i) Open Access}

\begin{abstract}
Objective: This study aimed to explore the relationship between traditional Chinese medicine (TCM) constitution and mental health levels of nursing staff in a general hospital. Methods: After random selection, 209 nursing staff were included to evaluate TCM constitution and mental health level through a TCM constitution checklist and the SCL-90. Results: 1) There were 102 cases (48.8\%) of balanced constitution and 107 cases of biased constitution (51.2\%). 2) The mental health status of the nursing staff was distributed as follows: suspected or mild (56.5\%), moderate (17.2\%), and severe (3.8\%). 3) In the correlation analysis between mental health status and TCM constitution, interpersonal sensitivity, phobia, and paranoia were negatively correlated with normal constitution and positively correlated with biased constitution. Conclusion: The mental health status of nursing staff is poor, and biased constitution may be a risk factor for their mental health. Intervention in biased constitutions may therefore improve the mental health of nursing staff.
\end{abstract}

\section{Keywords}

Nursing Staff, TCM Constitution, Mental Health, SCL-90

\section{Introduction}

Nursing staff are the frontline personnel in medical work. Their work is cumbersome and intensive. As a result, nursing staff can experience long-term chronic stress, and their physical and mental health face serious challenges. According to research reports outside of China, $28.8 \%-38.0 \%$ of nursing staff have 
psychological problems [1]. Research in China [2] [3], using the Symptom Checklist-90 (SCL-90) to evaluate nursing staff, has similarly found that the psychological status of nursing staff is generally lower than the national standard. This suggests that psychological problems are prevalent among the nursing population, which can easily develop into disease without timely intervention.

Since ancient times, traditional Chinese medicine (TCM) has focused on prevention prior to the onset of disease. TCM constitution theory suggests that TCM constitution is closely related to human health. Constitution is a comprehensive and relatively stable intrinsic trait in terms of morphological structure, physiological function, and mental state, formed on a congenital and acquired basis in human life. Constitution determines the body's susceptibility to certain pathogenic factors and tendency toward certain pathological types [4]. Therefore, constitution is a general term for the overall physical and mental quality of the human body, based on the integration of congenital and acquired traits. Constitution is relatively stable but also adjustable, and by adjusting constitution, the goal of preventing or effectively treating disease can be achieved.

Although TCM constitution is closely related to mental health, few studies have investigated this relationship. Therefore, this study conducted a sample survey of nursing staff in a hospital using the TCM constitution checklist and the SCL-90 to understand the relationship between TCM constitution and the mental health status of the nursing population. This research explores the relationship between TCM constitution and mental health and provides a reference for mental-illness prevention from the perspective of TCM constitution conditioning.

\section{Materials and Methods}

\subsection{Subjects}

The research subjects were recruited from a grade 3A hospital in Zhuhai, China. The inclusion criteria were as follows: 1) female, 2) nursing staff at the hospital, and 3) gave consent to participate in the study. The exclusion criteria were as follows: 1) had a previous mental illness such as depression, anxiety, or schizophrenia, 2) suffered from various organic diseases such as hypertension, diabetes, heart disease, or chronic kidney disease, 3) had major surgery or trauma in the past year, and 4) experienced serious life events in the past year, including divorce, becoming widowed, having a family member with a serious illness, losing a family member, suffering serious economic losses, or experiencing a major work accident. A random sampling method was used to extract 224 people from 747 frontline clinical nursing staff. The nursing staff were tested on the same day, and 209 valid questionnaires were collected (recovery rate: $93.3 \%)$.

\subsection{Research Methods}

The study was conducted in 2015. All subjects gave informed consent prior to 
participating. We conducted unified training before the survey and used unified standards and methods in the survey. The department heads of nursing staff in various departments were asked to distribute invitation kits containing explanatory notes, consent forms, three questionnaires, and a sealed envelope; these kits were randomly distributed to 224 nursing staff. Participants were asked to place the completed questionnaires and signed informed-consent forms in the sealed envelopes. The sealed envelopes were placed in a box at a designated location in the hospital that could only be opened by the researcher. After the questionnaires were collected, they were checked, and those that were unqualified (e.g., major items such as gender and age were missing, or there were several missing items) were excluded. The qualified questionnaires were entered into Epidata 3.1 Using a Double-Entry Method to Ensure Data Reliability.

\subsection{Research Tools}

\subsubsection{Self-Made Questionnaire}

We used a social demographics questionnaire that included gender, age, years of schooling, marital status, location of household registration, whether the participant was an only child, and personal history.

\subsubsection{TCM Constitution Identification Questionnaire}

The participants' constitution types were assessed according to the classification criteria of nine constitutions formulated by Wang Qi of the Beijing University of Chinese Medicine. The Checklist for the Classification and Judgment of TCM Constitution (also formulated by Wang Qi) was filled out by the nursing staff [4] [5] [6]. The checklist is a self-evaluation questionnaire consisting of nine subquestionnaires related to balanced constitution, qi deficiency, yang deficiency, yin deficiency, phlegm-dampness, blood stasis, qi stagnation, and special constitution (e.g., allergic). The conversion score of each subquestionnaire is 0 100 points. Among them, the eight constitution types other than normal constitution are all biased constitutions. Among these constitutions, balanced constitution is a normal constitution type, and the eight constitutions other than balanced constitution are all pathological constitution types.

The TCM constitutional judgment criteria are as follows. A person is considered to have a balanced constitution if the conversion score of the balanced constitution is $\geq 60$ points and the conversion scores of the other eight constitutions are $<30$ points. If the balanced constitution conversion score is $\geq 60$ points and the other eight constitutional scores are all $<40$ points, the person is considered to "basically" have a balanced constitution. For biased constitution, a conversion score of $\geq 40$ points means "yes," and a conversion score of 30 - 39 points means "likely." The higher the conversion score, the greater the tendency toward a certain constitution. This survey attributed a result of "basically" or "likely" to the corresponding constitution type. In this study, mixed constitutions were ignored, in order to only analyze the main effects. 


\subsubsection{Symptom Checklist 90 (SCL-90)}

The SCL-90, which is commonly used in China, was employed to test mental health status. The checklist consists of 90 items and covers a wide range of psychiatric symptoms. The checklist covers areas such as emotions, thoughts, behavior, lifestyle, interpersonal sensitivity, diet, and sleeping habits. The score ranges for the total symptom index are as follows: 1 - 1.5: subject experiences no symptoms listed in the checklist; 1.5 - 2.5: subject infrequently experiences mild symptoms; 2.5 - 3.5: subject experiences mild to moderate symptoms; 3.5 - 4.5: subject experiences moderate to severe symptoms; and 4.5 - 5: subject experiences symptoms frequently and with serious intensity.

\subsection{Statistical Analysis}

Statistical analyses were performed using SPSS 20.0. Counting data were analyzed using descriptive statistics. The relationship between constitution type and psychological symptoms was analyzed using Spearman's correlation analysis. $\mathrm{P}<$ 0.05 was considered statistically significant.

\section{Results}

\subsection{General Information}

All participants were female. They were $18-52$ years old (average: $28.5 \pm 7.56$ years), among which 84 participants $(40.2 \%)$ were $<25$ years old, $82(39.2 \%)$ were 25 - 35 years old, and 43 were 35 years old and above (20.6\%). In terms of education, 10 subjects had completed vocational high school (4.8\%), 83 completed junior college (39.7\%), 107 completed college (51.2\%), and 9 attended graduate school or above (4.3\%). A total of 125 participants were unmarried (59.8\%), and 84 participants were married (40.2\%). Details of general information are available in Table 1.

Table 1. General information, TCM constitution distribution characteristics, Mental health status in nursing staff.

\begin{tabular}{|c|c|c|}
\hline Variable & Number & Percentage \\
\hline \multicolumn{3}{|l|}{ Sex } \\
\hline $\operatorname{man}$ & 0 & 0 \\
\hline female & 209 & 100 \\
\hline \multicolumn{3}{|l|}{ Age } \\
\hline$\leq 25 \mathrm{y}$ & 84 & 40.2 \\
\hline $25-35 y$ & 82 & 39.2 \\
\hline$>35 y$ & 43 & 20.6 \\
\hline \multicolumn{3}{|l|}{ Education (years) } \\
\hline vocational high school & 10 & 4.8 \\
\hline junior college & 83 & 39.7 \\
\hline college & 107 & 51.2 \\
\hline graduate school or above & 9 & 4.3 \\
\hline
\end{tabular}




\section{Continued}

\begin{tabular}{|c|c|c|}
\hline \multicolumn{3}{|l|}{ Marital status } \\
\hline Unmarried & 125 & 59.8 \\
\hline Married & 84 & 40.2 \\
\hline \multicolumn{3}{|l|}{ TCM constitution } \\
\hline normal constitution & 102 & 48.8 \\
\hline biased constitution & 107 & 51.2 \\
\hline qi stagnation & 32 & 15.3 \\
\hline damp-heat & 16 & 7.7 \\
\hline qi deficiency & 16 & 7.7 \\
\hline yang deficiency & 15 & 7.2 \\
\hline blood stasis & 9 & 4.3 \\
\hline phlegm-dampness & 8 & 3.8 \\
\hline special & 8 & 3.8 \\
\hline yin deficiency & 5 & 2.4 \\
\hline \multicolumn{3}{|l|}{ Mental health } \\
\hline Suspicious or mild cases accounted & 118 & 56.5 \\
\hline moderate & 36 & 17.2 \\
\hline severe & 8 & 3.8 \\
\hline \multicolumn{3}{|l|}{ Abnormal rate of Mental health } \\
\hline Obsession & 145 & 69.4 \\
\hline Interpersonal sensitivity & 115 & 55 \\
\hline Depression & 89 & 42.6 \\
\hline Hostility & 73 & 34.9 \\
\hline Anxiety & 67 & 32.1 \\
\hline Psychosis & 64 & 30.6 \\
\hline Paranoia & 61 & 29.2 \\
\hline Phobia & 49 & 23.4 \\
\hline Somatization & 49 & 23.4 \\
\hline
\end{tabular}

\subsection{TCM Constitution Distribution Characteristics}

According to the TCM constitution checklist, the ratio of normal constitution to biased constitution was 1:1.05. Among the nine different constitutions, the most common was normal constitution (102 cases, 48.8\%). There were 107 cases of biased constitution, accounting for $51.2 \%$; these cases were distributed as follows: qi stagnation $(\mathrm{n}=32,15.3 \%)$, damp-heat $(\mathrm{n}=16,7.7 \%)$, qi deficiency $(\mathrm{n}=$ $16,7.7 \%)$, yang deficiency $(\mathrm{n}=15,7.2 \%)$, blood stasis $(\mathrm{n}=9,4.3 \%)$, phlegm-dampness $(n=8,3.8 \%)$, special $(n=8,3.8 \%)$, and yin deficiency $(n=5$, $2.4 \%)$.

\subsection{Mental Health Status}

A total of 209 nursing staff were evaluated by the SCL-90. If one factor in the evaluation result exceeded the normal level, the result was considered abnormal; suspicious or mild cases accounted for $56.5 \%$, and moderate to severe cases ac- 
counted for $21.0 \%$ of the total. From high to low, the abnormal factors included compulsion $(n=145,69.4 \%)$, interpersonal sensitivity $(n=115,55.0 \%)$, depression $(n=89,42.6 \%)$, hostility $(n=73,34.9 \%)$, anxiety $(n=67,32.1 \%)$, psychosis $(\mathrm{n}=64,30.6 \%)$, paranoia $(\mathrm{n}=61,29.2 \%)$, phobia $(\mathrm{n}=49,23.4 \%)$, and somatization $(\mathrm{n}=49,23.4 \%)$.

\subsection{Relationship between Constitution Types and Psychological Symptoms}

Spearman's correlation analysis between constitution types and psychological symptoms indicated a negative correlation between normal constitution and interpersonal sensitivity, phobia, and paranoia. A positive relationship was indicated between biased constitution and compulsion, interpersonal sensitivity, phobia, and paranoia. Details of the relevant analysis are available in Table 2.

\section{Discussion}

\subsection{Relationship between TCM Constitution and Physical Health}

In TCM [5], constitution is an objective life phenomenon as well as a carrier of health and disease. Constitution type affects an individual's predisposition to disease and the symptoms after its onset. A biased constitution is the basis for the occurrence of related diseases. Before the onset of disease, adjusting a biased constitution can improve an individual's health. Such adjustment can also prevent the occurrence of related diseases and achieve the goal of health management. The proportion of balanced constitution to biased constitution among the participants was 1:1.05. Among the eight biased constitutions, qi stagnation was the most common (15.3\%), followed by damp-heat (7.7\%), qi deficiency (7.7\%), and yang deficiency $(7.2 \%)$. The proportion of biased constitutions among the nursing staff was more than $50 \%$, suggesting that the constitution distribution of the nursing staff is poor, which may suggest that the nursing population is more susceptible to diseases.

Table 2. Relationship between TCM constitution types and psychological symptoms among nursing staff ( $r$-values).

\begin{tabular}{|c|c|c|c|c|c|c|c|c|c|}
\hline Item & Somatization & Coercion & $\begin{array}{l}\text { Interpersonal } \\
\text { sensitivity }\end{array}$ & Depression & Anxiety & Hostility & Phobia & Paranoia & Psychosis \\
\hline Normal constitution & 0.028 & -0.156 & $-0.180^{*}$ & -0.35 & 0.017 & -0.016 & $-0.188^{*}$ & $-0.219^{* *}$ & -0.018 \\
\hline Constitution of qi deficiency & 0.036 & $0.165^{\star}$ & 0.125 & 0.029 & 0.040 & 0.055 & 0.151 & 0.153 & 0.014 \\
\hline $\begin{array}{l}\text { Constitution of yang } \\
\text { deficiency }\end{array}$ & 0.081 & 0.147 & $0.161^{\star}$ & 0.095 & 0.064 & 0.028 & 0.145 & $0.182^{*}$ & 0.023 \\
\hline Constitution of yin deficiency & 0.028 & $0.179^{*}$ & 0.154 & 0.070 & 0.125 & 0.000 & 0.118 & $0.163^{\star}$ & 0.095 \\
\hline $\begin{array}{l}\text { Constitution of } \\
\text { phlegm-dampness }\end{array}$ & -0.013 & 0.124 & 0.099 & -0.002 & 0.061 & -0.022 & 0.083 & 0.126 & 0.023 \\
\hline Constitution of damp-heat & -0.036 & 0.123 & 0.120 & 0.016 & 0.086 & -0.072 & 0.116 & 0.153 & 0.095 \\
\hline Constitution of blood stasis & -0.014 & $0.163^{*}$ & 0.128 & 0.060 & 0.054 & 0.043 & $0.160^{*}$ & $0.234^{\star *}$ & 0.072 \\
\hline Constitution of qi stagnation & 0.027 & $0.183^{*}$ & 0.141 & 0.062 & 0.070 & 0.069 & $0.191^{*}$ & $0.199^{*}$ & 0.056 \\
\hline Special constitution & 0.071 & 0.125 & 0.104 & 0.068 & 0.044 & 0.007 & 0.098 & 0.148 & 0.120 \\
\hline
\end{tabular}


Research on constitution and suboptimal health in different regions [7] [8] [9] [10] found that people with suboptimal health had higher proportions of damp-heat and qi-deficiency constitution. Ou et al. [10] investigated populations in Guangdong, Beijing, Shanghai, and Chengdu and found that suboptimal physical health corresponded to yang and yin deficiency, while suboptimal mental health corresponded to qi deficiency and blood stasis. The present study found that among biased constitutions, qi stagnation occurred most frequently, followed by damp-heat, qi deficiency, and yang deficiency, suggesting that the physical and mental health of the investigated nursing staff was not good. Qi stagnation [6] forms as a result of long-term emotional discomfort; the personality is introverted, unstable, depressed, sensitive, and suspicious. A person with qi stagnation may also have various physical discomforts such as chest tightness, belching or hiccups, foreign-body sensation in the throat, insomnia, frequent dreams, and loss of appetite, as well as mental issues including depression, anxiety, and neurasthenia [6]. Xiong [11] conducted a clinical epidemiological survey of TCM constitutions in the Taipei area and found that individuals with qi stagnation were more likely to have borderline depression than those with balanced constitution. Furthermore, qi stagnation was accompanied by a high probability of major depression. The present research found that qi stagnation was the most common biased constitution among the nursing staff in the studied hospital. We speculate that this result could be related to high work stress, interpersonal sensitivity, and long-term depression, suggesting that without intervention, the risk of depression is likely to increase.

\subsection{Relationship between TCM Constitution and Mental Health}

Research outside of China [1] has revealed that $28.8 \%-38.0 \%$ of nursing staff have psychological problems. The present study found mild-to-severe psychological problems among $21 \%$ of the investigated nursing staff. Numerous studies [12] [13] [14] have found that TCM constitution is closely related to mental health. However, people with different constitutions have different levels of tolerance for stressful events. For example, individuals with normal constitutions are better able to adjust to stressful events because of their stable personalities. Meanwhile, those with biased constitutions are often more fragile and have weak psychological tolerance. These individuals easily experience negative emotions such as anxiety, depression, phobia, or hostility, which in turn give rise to mental illness. Zhu et al. [15] studied a general population of 8848 people in China and found that those with normal constitutions had better mental health than those with biased constitutions. In the biased-constitution population, qi stagnation showed the lowest score among the three dimensions of social function, emotional function, and mental health. Females with qi stagnation scored the lowest in the two dimensions of emotional function and mental health. Xu et al. [13] surveyed 1032 college students and found that qi deficiency was related to interpersonal sensitivity while qi stagnation was related to depression. Similarly, the present study found a strong negative correlation between a normal consti- 
tution and interpersonal sensitivity, phobia, paranoia, and psychosis, whereas there was a strong positive correlation between biased constitutions and compulsion, interpersonal sensitivity, phobia, and paranoia. In the TCM constitution checklist, the higher the score for each biased-constitution factor, the more typical the manifestation of that constitution. On the SCL-90, the higher the score for each factor, the more serious the psychological symptoms. Therefore, the more the participants tended to have biased constitutions, the worse their mental health status. Therefore, we concluded that the mental health of the nursing staff was closely associated with having a biased constitution, which is a risk factor for mental health.

In summary, the proportion of nursing staff with biased constitutions was relatively high, and their level of mental health was relatively low. Correlation and multiple regression analyses indicated that factors such as compulsion, interpersonal sensitivity, phobia, and paranoia were closely related to biased constitutions, suggesting that using TCM and other means to transform biased constitutions into balanced constitutions may improve the physical and mental health of nursing staff and thus reduce the occurrence of disease.

\subsection{This Study Has Some Limitations}

The number of nursing staff included in the study was fairly small, and participants with certain constitutions accounted for relatively small proportions, such as blood stasis (4.3\%), phlegm-dampness (3.8\%), special constitution (3.8\%), and yin deficiency $(2.4 \%)$. Therefore, the sample size should be expanded to further clarify which constitutions are associated with which mental health issues. In addition, we did not distinguish different departments and therefore did not consider the different working conditions and work pressures nursing staff might face in different departments. We also did not address the effect of working night shifts. Previous studies have found that [16] working night shifts can adversely affect the mental health of nursing staff. Moreover, we did not investigate the coping styles and personality traits of the nursing staff, which may affect mental health [17]. Lastly, this was a cross-sectional study with no intervention for the nursing staff. In future research, the authors will expand the sample size, include more observation factors (e.g., night shift, personality traits, coping styles), and increase interventions to further study the relationship between TCM constitution and mental health.

\section{Acknowledgements}

We would like to thank all the participants for their contribution to this study: Xiaojuan Fang, Minfeng Cheng.

\section{Conflicts of Interest}

The authors declare no conflicts of interest regarding the publication of this paper. 


\section{References}

[1] Petterson, I.L., Arnetz, B., Arnetz, J.E., et al. (1995) Work Environment, Skills Utilization and Health of Swedish Nurses-Results from a National Questionnaire Study. Psychotherapy and Psychosomatics, 64, 20-31. https://doi.org/10.1159/000288987

[2] Zhu, S.H., Luo, H., Ji, Y., et al. (2009) Study on the Relationship between Nurses' Psychological Health and Coping Style in General Hospital. Modern Preventive Medicine, 36, 2488-2489.

[3] Diao, L.H., Guo, Y. and Chen, D.Z. (2004) Advance in Safeguarded Mental Health of Nurse. Chinese Journal of Nursing, No. 8, 55-58.

[4] TCM Constitution Classification and Judgment (2009) ZYYXH/T157-2009. World Journal of Integrated Traditional Chinese and Western Medicine, No. 4, 303-304.

[5] Wang, Q. (2012) Research and Application of Constitution Theory in Traditional Chinese Medicine. China Traditional Chinese Medicine Press, Beijing.

[6] Wang, Q. (2005) Classification and Diagnosis Basis of Nine Basic Constitutions in Chinese Medicine. Journal of Beijing University of Traditional Chinese Medicine, No. 4, 1-8.

[7] Huang, Y., Huang, Y., Zhang, T.J., et al. (2012) Chinese Medicine Identification and Effective Factors Analysis in Sub-Healthy People. Journal of Emergency in Traditional Chinese Medicine, No. 2, 178-179.

[8] Tian, S., Qi, R.K. and Cheng, Y.Z. (2015) Preliminary Study on Charastics and the Relationship between the TCM Constitutions and Syndrome Elements of Five Hundred and Fourteen Cases of Sub-Health Population. China Journal of Traditional Chinese Medicine and Pharmacy, No. 1, 243-245.

[9] Bao, W.T. and Tang, J. (2014) Investigation and Analysis of Chinese Medicine Constitutions Identification among Sub-Health of 689 Cases. China Modern Doctor, No. 24, 116-118.

[10] Ou, A.H., Mai, R.R., Yuan, J.M., et al. (2012) The Correspondence Analysis of the Relationship between Sub-Health Condition Typing and Constitutions of TCM. Guangdong Medical Journal, No. 1, 11-14.

[11] Xiong, L. (2007) Study on the Constitution of QI Stagnation and Its Distribution in Taipei. Beijing University of Chinese Medicine, Beijing.

[12] Xue, L.F. (2014) Correlation Research for Traditional Chinese Medicine Syndrome Type and Constitution of Senile Depression. Hebei Journal of Traditional Chinese, No. 9, 1303-1307.

[13] Xu, Y.H. and Wang, Z.Z. (2012) Correlation between TCM Constitution and Psych Symptoms in College Students. Guiding Journal of Traditional Chinese Medicine and Pharmacology, No. 12, 21-23.

[14] Zhong, M.F., Huang, X.Q., Wei, Y.Y., et al. (2017) A Preliminary Study on the Feasibility of Intervention of Mental Health by Traditional Chinese Medicine Constitution Theory. Psychological Exploration, No. 5, 392-395.

[15] Zhu, Y.B., Wang, Q., Chen, K.P., et al. (2011) Stratified Analysis of the Relationship between Traditional Chinese Medicine Constitutional Types and Health Status in the General Population Based on Data of 8448 Cases. Journal of Chinese Integrative Medicine, No. 4, 382-389.

[16] Jiang, Y., Zhang, Y., Deng, X.Y., et al. (2012) Mental Condition of Emergency Nurse and Coping Style. China Journal of Health Psychology, 20, 1348-1349. 
[17] Tan, J.C. (2014) Study on the Relationship between TCM Constitution Type, Personality Psychology and Sleep Quality in College Students. Guangzhou University of Chinese Medicine, Guangzhou. 